\title{
Pediatric Hematopoietic Cell Transplantation: Longitudinal Trends in Body Mass Index and Outcomes
}

\author{
Brian Dang ${ }^{1}$, Holly Wilhalme ${ }^{2}$, James Ch'ng ${ }^{1}$, Satiro De Oliveira ${ }^{3}$, Lavette Bowles ${ }^{1}$, and \\ Ted Moore ${ }^{4}$ \\ ${ }^{1}$ University of California Los Angeles David Geffen School of Medicine \\ ${ }^{2}$ Division of General Internal Medicine and Health Services Research, University of \\ California Los Angeles \\ ${ }^{3}$ University of California \\ ${ }^{4}$ UCLA Medical Center
}

June 2, 2020

\begin{abstract}
Background Obesity is an increasing problem in the United States, with one in five adolescents obese. Past studies have shown that pediatric recipients of hematopoietic cell transplants (HCT) may have a higher susceptibility to overweight and obesity. Procedure This is a single-center retrospective analysis of 297 pediatric patients who received HCT between 2005-2018. Patients were divided into four weight categories of underweight (UW), normal weight (NW), overweight (OW), and obese (OB) based on age adjusted body mass index (BMI) conversion scales. Post-transplant outcomes included acute graft vs. host disease (GVHD), chronic graft vs. host disease, viral infection rates, time to platelet and neutrophil engraftment, and overall survival. Results In the pre-transplant period, the percentage of individuals who were UW, NW, OW, and OB were $5.4 \%, 54.5 \%, 22.2 \%$, and $17.8 \%$ respectively. At the five-year post-transplant mark, those numbers were $10.6 \%, 48.2 \%, 16.5 \%$, and $24.7 \%$. Overall, BMI was found to increase $0.00094 \pm 0.0001 \mathrm{~kg} / \mathrm{m} 2$ each day after transplant $(\mathrm{p}<0.001)$, with older individuals demonstrating greater trends of BMI increase. Further, there was a larger BMI increase in those who did not receive TBI compared with those who received TBI $(1.29 \pm 0.49, \mathrm{p}=0.008)$. The rates of acute graft vs. host disease (GVHD), chronic GVHD, and viral infection, in addition to time to platelet and neutrophil engraftment and 5-year survival estimate, were not significantly different among weight groups. Conclusion Pediatric HCT recipients are at high risk of developing overweight or obesity after transplant.
\end{abstract}

\section{Pediatric Hematopoietic Cell Transplantation: Longitudinal Trends in Body Mass Index and Outcomes}

Running Title: Pediatric hematopoietic cell transplant obesity

Brian Norman Dang, BS ${ }^{1}$, Holly Wilhalme, MS², James Ch'ng, MD¹, Satiro De Oliveira, MD', LaVette Bowles, $\mathrm{NP}^{1}$, Theodore Bruce Moore, $\mathrm{MD}^{1}$

${ }^{1}$ Division of Pediatric Hematology and Oncology, University of California Los Angeles David Geffen School of Medicine, Los Angeles, California

${ }^{2}$ Division of General Internal Medicine and Health Services Research, University of California Los Angeles

Corresponding Author:

Theodore B. Moore, MD

Professor of Pediatrics; 
Clinical Director, Pediatric Hematology/Oncology

Director, Pediatric Blood and Marrow Transplant Program

UCLA Mattel Children's Hospital

10833 Le Conte Ave, MDCC A2-410

Los Angeles, CA, USA 90095

Phone: +1 (310) 825-6708

Fax: +1 (310) 206-8089

bdang@mednet.uca.edu

Abstract Word Count: 242

Main Text Word Count: 2896

\# Figures/Tables: 3 tables, 2 figures

Keywords: pediatric, hematopoietic cell transplant, body mass index, obesity

\begin{tabular}{ll}
\hline Abbreviation & Full term \\
\hline ALL & acute lymphoblastic leukemia \\
AML & acute myeloid leukemia \\
BMI & body mass index \\
GVHD & graft vs host disease \\
HCT & hematopoietic cell transplant \\
HR & hazard ratio \\
NW & normal weight \\
OB & obese \\
OW & overweight \\
TBI & total body irradiation \\
UW & underweight \\
WHO & world health organization \\
\hline
\end{tabular}

\section{Abstract}

\section{Background}

Obesity is an increasing problem in the United States, with one in five adolescents obese. Past studies have shown that pediatric recipients of hematopoietic cell transplants (HCT) may have a higher susceptibility to overweight and obesity.

\section{Procedure}

This is a single-center retrospective analysis of 297 pediatric patients who received HCT between 2005-2018. Patients were divided into four weight categories of underweight (UW), normal weight (NW), overweight $(\mathrm{OW})$, and obese (OB) based on age adjusted body mass index (BMI) conversion scales. Post-transplant outcomes included acute graft vs. host disease (GVHD), chronic graft vs. host disease, viral infection rates, time to platelet and neutrophil engraftment, and overall survival.

\section{Results}

In the pre-transplant period, the percentage of individuals who were $\mathrm{UW}, \mathrm{NW}$, OW, and $\mathrm{OB}$ were $5.4 \%$, $54.5 \%, 22.2 \%$, and $17.8 \%$ respectively. At the five-year post-transplant mark, those numbers were $10.6 \%$, 
$48.2 \%, 16.5 \%$, and $24.7 \%$. Overall, BMI was found to increase $0.00094 \pm 0.0001 \mathrm{~kg} / \mathrm{m}^{2}$ each day after transplant $(p<0.001)$, with older individuals demonstrating greater trends of BMI increase. Further, there was a larger BMI increase in those who did not receive TBI compared with those who received TBI (1.29 $\pm 0.49, \mathrm{p}=0.008)$. The rates of acute graft vs. host disease (GVHD), chronic GVHD, and viral infection, in addition to time to platelet and neutrophil engraftment and 5-year survival estimate, were not significantly different among weight groups.

\section{Conclusion}

Pediatric HCT recipients are at high risk of developing overweight or obesity after transplant.

\section{Introduction}

Obesity is an increasing problem in the United States, with one in five adolescents obese, ${ }^{1}$ and a leading cause of morbidity and mortality. The negative consequences of obesity on health has been well documented in the literature, ${ }^{2,3}$ prompting clinical and public health measures for both treatment and prevention. ${ }^{4}$ Further, certain individuals, such as survivors of childhood malignant diseases including acute lymphoblastic and myeloid leukemias (ALL and AML) have demonstrated increased risk for obesity ${ }^{5-8}$ and cardiometabolic disease ${ }^{9-11}$ in comparison to their corresponding age group in the general population. This increased susceptibility for weight gain has been noted early in treatment, throughout treatment, and well beyond. ${ }^{12}$

Hematopoietic cell transplantation (HCT) is a well-established and widely utilized treatment for a variety of malignant and non-malignant conditions. ${ }^{13}$ Efforts to better understand and predict the impacts of comorbidities on transplant outcomes have led to the creation of the HCT comorbidity index, which includes obesity as one of the factors for risk assessment and prediction of nonrelapse mortality and survival. ${ }^{14}$ With recent studies demonstrating an association between obesity and reduced survival in adult ${ }^{15}$ and pediatric ${ }^{16}$ HCT recipients, the growing interest on body habitus, a potentially modifiable risk factor, and its effects on HCT outcomes have prompted research on the converse - the influence of HCT on body mass index (BMI). Thus far, the literature has demonstrated that pediatric HCT recipients may experience a reduction in BMI after transplantation, ${ }^{17}$ as well as an increase. ${ }^{18}$ As the data on the weight status of children after HCT is limited and inconsistent, further exploration in this field is needed.

In this study, we sought to characterize the interactions between pediatric BMI and HCT outcomes at our institution. We have included longitudinal data during the pre-transplant period up to 5 years posttransplantation, to investigate the trends in weight status and outcomes throughout the pre-transplant, immediate post-transplant, and long-term post-transplant periods.

\section{Materials and Methods}

\section{Patient Characteristics and Outcomes Measured}

We conducted a retrospective analysis of HCT patients treated at Mattel Children's Hospital at the University of California, Los Angeles. This study was approved by the UCLA institutional review board (IRB \# 17001107). All patients who received HCT over a 14-year period between 2005 and 2018 were included in this study.

Data on diagnosis, age at transplant, sex, ethnicity, malignant, relationship to donor, stem cell source, antigen mismatch, and conditioning regimen with total body irradiation (TBI) were collected. Post-transplant followup data included acute graft vs. host disease (GVHD) within the first 100 days, chronic GVHD after 100 days and use of steroids, rates of viral infection, time to platelet and neutrophil engraftment, and overall survival.

\section{Anthropometry}

BMI (measured in $\mathrm{kg} / \mathrm{m}^{2}$ ) was evaluated from baseline (time of transplant) to up to five years post-transplant. Height and weight data were collected during clinic visits using a metric scale and wall mounted stadiometer. BMI from patients less than 20 years old were converted to percentiles using age appropriate scales. For 
patients younger than 2 years, the "Child Growth Standards" SAS macro supplied by the World Health Organization (WHO) was used. ${ }^{19}$ For patients 2 - 20 years old, we used an SAS program from the Centers for Disease Control and Prevention. ${ }^{20}$ Percentiles were stratified to weight groups according to the following: obese $(>95 \%)$, overweight $(85-94.9 \%)$, normal weight $(5-84.9 \%)$, and underweight $(<5 \%)$. For patients older than 20 years old, we used the WHO criteria for adults ${ }^{21}$ to stratify BMI according to the following: obese $\left(>30 \mathrm{~kg} / \mathrm{m}^{2}\right)$, overweight $\left(25-29.9 \mathrm{~kg} / \mathrm{m}^{2}\right)$, normal weight $\left(18.5-24.9 \mathrm{~kg} / \mathrm{m}^{2}\right)$, and underweight $(<$ $\left.18.5 \mathrm{~kg} / \mathrm{m}^{2}\right)$.

\section{Statistical Analyses}

Patient demographics and clinical characteristics were summarized by weight category using means and standard deviations for continuous variables and frequencies and percentages for categorical variables.

A mixed effects linear regression model was used to determine if there was a significant change from baseline BMI over time among the cohort. This model included a fixed effect for BMI and a random effect for the patient. Covariates included in the model were patient baseline age at time of transplant, antigen mismatch, sex, relapse, malignant, ethnicity, TBI, and transplant source. Further, the relationship between the change in BMI over time and age was explored by including an interaction term between age and time.

Fisher's exact test was used to determine if there were significant differences in the proportion of patients with GVHD (chronic and acute) and viral infections between the pre-transplant weight categories. The Kaplan-Meier method was used to estimate the median time to neutrophil and platelet engraftment and the overall survival rates at 5 years based on pre-transplant weight category.

Cox proportional hazards models were utilized to estimate the hazard ratio (HR) for overall survival, in addition to times to platelet engraftment and neutrophil engraftment, for patients in the overweight and obese pre-transplant weight categories versus patients in the normal weight/underweight pre-transplant weight category. Models were adjusted for age, transplant source, and mismatch. For overall survival, if patients did not die, they were censored at the time the data was pulled (i.e. December 31, 2019). For the analyses of time to neutrophil engraftment and time to platelet engraftment, a cause-specific hazards regression model was used to account for the competing risk of death for subjects who died before they could engraft. Patients were excluded if they were missing engraftment data due to death or transfer of care prior to engraftment. All HRs were accompanied by their corresponding 95\% confidence interval (CI). SAS Version 9.4 (Cary, NC USA) was used for all statistical analyses. A p-value $<0.05$ was considered statistically significant.

\section{Results}

\section{Patient Characteristics}

The demographics and clinical characteristics were collected for the 297 patients who were included in this study (Table 1). The average age at transplant was 8.4 years. Two-thirds of the patients were male $(66.7 \%)$ and the majority were non-Caucasian (65.3\%). Roughly half $(56.6 \%)$ of the individuals received a 6/6 HLA matched transplant, and $68.4 \%$ were from an allogenic source. The majority of patients $(78.8 \%)$ did not receive TBI for their conditioning regimen. The majority of patients had transplants for malignant diseases $(65 \%)$.

\section{BMI trends}

The frequencies of BMI categories were compared at baseline, 100 days post-transplant, and at one-year time intervals until five years post-transplant. In the pre-transplant period, the percentage of individuals who were underweight $(\mathrm{UW})$, normal weight $(\mathrm{NW})$, overweight $(\mathrm{OW})$, and obese $(\mathrm{OB})$ were $5.4 \%, 54.5 \%$, $22.2 \%$, and $17.8 \%$ respectively (Table 2 ). At the five-year post-transplant mark, those numbers were $10.6 \%$, $48.2 \%, 16.5 \%$, and $24.7 \%$, respectively.

The mixed effects linear regression model demonstrated a significant change from baseline BMI over time after controlling for age at transplantation, mismatch, sex, relapse, malignant, ethnicity, TBI, and transplant 
source, where BMI was found to increase $0.00094 \pm 0.0001 \mathrm{~kg} / \mathrm{m}^{2}$ each day after transplant $(p<0.001)$. In addition, TBI was significant, such that there was greater change in BMI from baseline in those who did not receive TBI compared with those who received TBI $(1.288 \pm 0.496, p=0.010)$.

The inclusion of an interaction term between age and time further demonstrated distinct trends. Effects were estimated for the average 6-month-old, 2-year-old, 7-year-old, 12-year-old, and 16-year-old. While all patients overall experienced an increase in BMI over time that was more pronounced in older age groups (interaction effect between BMI and time: $0.000075 \pm 0.00002, p<0.001$ ), a drop in BMI in the first 100 days post-transplant, though not significant, was observed in the 7-year, 12-year, and 16-year old (Figure 1). Patients 6 months and 2 years of age demonstrated an increase in BMI within the first 100 days posttransplant that was not statistically significant. After 100 days post-transplant, all age groups demonstrated significant increases in BMI measured per 100-day increment. These trends of increasing BMI relative to baseline, starting from day 100 to 5 years, were more drastic with each older age group.

\section{Post-Transplant Outcomes}

The post-transplant outcomes were compared across weight categories. The rates of mild acute GVHD (grade $0-2$ ) were $83.3 \%, 83.5 \%, 84.4 \%$, and $74.4 \%$ and the rates of severe acute GVHD (grade $3-4$ ) were $16.7 \%, 16.5 \%$, $15.6 \%$, and $25.6 \%$ for individuals in the $\mathrm{UW}, \mathrm{NW}, \mathrm{OW}$, and $\mathrm{OB}$ weight categories respectively $(p=0.54)$ (Table 3). The rates of 0-limited chronic GVHD were $83.3 \%, 92.2 \%, 88.1 \%$, and $94.4 \%$, while the rates for moderate-severe chronic GVHD were $16.7 \%, 7.8 \%, 11.9 \%$, and $5.6 \%$ for individuals in the UW, NW, OW, and $\mathrm{OB}$ weight categories respectively $(p=0.48)$. Individuals in the UW, NW, OW and OB weight categories did not demonstrate statistically significant differences in rates of viral infections $(p=0.37)$, time to platelet engraftment $(p=0.99)$, time to neutrophil engraftment $(p=0.88)$, or 5 -year survival $(p=0.32)$.

Cox proportional hazards models estimated the hazard ratios for engraftment and overall survival. The hazard ratios for time to platelet engraftment were not significantly different when comparing patients in the OW category (HR 1.325, 95\% CI $0.883-1.986, p=0.174$ ) and OB category (HR $1.205,95 \%$ CI 0.799 $-1.818, p=0.374$ ) versus individuals in the NW/UW category. The hazard ratios for time to neutrophil engraftment were not significantly different when comparing patients who were OW (HR 1.238, 95\% CI $0.885-1.731, p=0.213$ ) and OB (HR $0.878,95 \%$ CI $0.593-1.298, p=0.513$ ) with NW/UW patients. The hazard ratios for overall survival for individuals in the OW weight category (HR 1.317, 95\% CI 0.739 $-2.348, p=0.351$ ) and OB category (HR 1.072, 95\% CI $0.576-1.994, p=0.827$ ) showed no significant differences compared with those who were NW/UW. The Kaplan-Meier plot of overall survival probability showed trends of worsened survival in OW and OB individuals compared with NW/UW individuals, though not significant $(p=0.377)$ (Figure 2).

\section{Discussion}

Obesity represents a growing health risk in this country. Pediatric patients with hematopoietic malignancies are no exception. In this study, we demonstrate that pediatric patients experience an increase in BMI after HCT. Prior to transplant, $40 \%$ of patients were overweight or obese according to BMI, of which $17.8 \%$ qualified as obese. At the five years post-transplant mark, $40.5 \%$ were overweight or obese, of which $24 \%$ were obese. While the total number of overweight and obese remained constant, the number of obese patients increased, likely secondary to overweight individuals becoming obese. Further, our longitudinal analysis of BMI trends over time demonstrated the highest rate of BMI increase in older patients. In the general US pediatric population, the prevalence of overweight and obesity is $31.8 \%$, with $16.9 \%$ obese. Similarly, the prevalence is higher in older youth aged $12-19$, with $34.5 \%$ overweight or obesity, of which $20.5 \%$ classify as obese. ${ }^{22}$ In Los Angeles County, $23.0 \%$ of school-aged children are obese. ${ }^{23}$ Despite the full-time support of a registered dietician in both the inpatient and outpatient settings for the UCLA pediatrics hematology and oncology service, our cohort demonstrated similar rates of obesity compared with children in Los Angeles county, and higher rates of obesity compared with the general US pediatric population. This suggests that pediatric HCT patients have a high predisposition for obesity in the years following transplantation, with adolescents/young adults at highest risk. 
Understanding the trends in BMI after HCT is critical, yet few longitudinal reports of body composition in HCT patients exist and the results are inconsistent. A European report characterized the trends in BMI for both adult and pediatric patients after $\mathrm{HCT}$, noting that BMI remained stable in adults while it increased in children. ${ }^{24}$ However, this study did not analyze BMI using age-appropriate scales in children. A more recent report adjusted BMI utilizing the same age conversion scales as we did. This study of 179 HCT recipients over a 10 year span demonstrated a drop in BMI after transplantation due to a reduction in lean mass. ${ }^{17}$ Conversely, it has been shown that childhood HCT recipients are at risk of developing central obesity that is not adequately captured through BMI measures. ${ }^{18}$ Our work contributes to this understudied topic, suggesting that childhood HCT survivors experience weight gain that disproportionately affects older patients.

There are several reasons that may contribute to this increased predisposition for obesity. Complications after HCT, such as GVHD, are frequently treated with steroids that can cause significant toxicity including weight gain and central obesity. ${ }^{25}$ Sleep disruption is also common among HCT recipients, ${ }^{26}$ which may influence weight status. ${ }^{27} \mathrm{HCT}$ recipients may experience physical fatigue and poor functional status that can persist for several years post-transplantation. ${ }^{28}$ Further, chemotherapy can induce endothelial damage, causing capillary leakage and fluid retention. ${ }^{29}$ While not all of these directly reflect nutritional status and degree of adiposity, indirect and direct factors that promote fatty deposition and sedentary lifestyles are concerning. Excessive weight and obesity induce a chronic low grade inflammatory state that predisposes to a wide variety of conditions, including metabolic syndrome. ${ }^{30}$ The impacts of obesity on HCT is complex, such that excess adiposity may directly affect disease pathogenesis and alter pharmacodynamics, further complicated by the often varying conditioning regimens for those who are obese. ${ }^{31}$ While a consensus has not been reached regarding the effects of obesity on HCT outcomes, many studies highlight its negative effects. One report in both children and adults receiving HCT demonstrated higher rates of non-relapse related mortality in obese patients, likely due to acute and chronic GVHD, although with no difference in overall survival. ${ }^{32}$ A meta-analysis found that a high pre-transplant BMI was associated with an increased risk for acute GVHD along with worsened survival. ${ }^{33}$ In the pediatric literature, two studies revealed that high BMI pre-transplantation led to lower overall survival and higher mortality. ${ }^{16,34}$ While our post-transplant outcomes analysis regarding GVHD, infection, platelet and neutrophil engraftment, and overall survival were not statistically significant among weight categories, our results do show trends towards higher rates of severe acute GVHD and lower survival for overweight and obese patients. Thus, further research is warranted to investigate the impacts of obesity on HCT outcomes.

A notable trend in our analysis of BMI is that those who did not receive TBI had a higher BMI increase from baseline, such that TBI demonstrated a protective effect in weight gain. TBI may induce hypothalamic pituitary lesions that disrupt the regulatory secretion of hormones that contribute to height and nutritional status. ${ }^{35}$ Further, it has been demonstrated that TBI may decrease GH secretion, interrupt leptin regulation, and result in a persistently low BMI and blunted adult height. ${ }^{36,37}$ We believe that the trends in our weight analysis may be the result of similar hypothalamic disruptions, where TBI may hinder growth in both height and weight that precludes a BMI increase.

Regardless of the data on HCT outcomes, it is well established that obesity is associated with a variety of comorbidities. Chronic adaptations in cardiac structure and function in response to excess adipose tissue accumulation increases risk of cardiovascular disease, which can manifest as heart failure, coronary heart disease, and sudden cardiac death. ${ }^{38,39}$ Given that many HCT patients receive doxorubicin, an anthracycline with severe cardiotoxic side effects, ${ }^{40}$ it is especially critical to optimize body composition in this vulnerable population. A proactive approach by maintaining a healthy BMI before treatment, and well after, may mitigate the risk of adverse cardiovascular events later in life.

The idea that there is a close relationship among nutrition, HCT, and oncology is not novel. Since the observation of the Warburg effect, ${ }^{41}$ the literature has expanded with attempts to better understand the relationship between nutrition and tumorigenesis. There currently exists several proposed dietary regimens, including the ketogenic diet ${ }^{42}$ intermittent fasting, ${ }^{43}$ and caloric restriction ${ }^{44}$ as emerging approaches in 
cancer treatment. It is believed that an avoidance of the Western diet, ${ }^{45}$ and an adaptation of the Japanese diet, ${ }^{46}$ vegan diet, ${ }^{47}$ or Mediterranean $\operatorname{diet}^{48}$ may decrease risk of developing a wide variety of malignancies. Due to the possible influences of nutrition on tumorigenesis and transplant outcomes, optimization of body composition throughout the process of HCT is essential. ${ }^{49}$

This is a comprehensive analysis of the HCT data from the pediatric population at our institution. Nonetheless, our study, which is retrospective in nature, is limited by the availability of patient data. Thus, we were only able to include patients who received HCT from 2005 to 2018 since those who were transplanted prior to 2005 had incomplete clinical data that were insufficient to include in our study. Further, there may be potential confounders in the patient characteristics affecting post-transplant outcomes; however, these do not affect our overall observation of increased BMI after HCT. Another limitation is our reliance on BMI to assign patients into the appropriate weight categories. There is evidence to suggest that BMI may not adequately represent body fat percentage and those who have central obesity. ${ }^{18}$ Unfortunately, data on body fat percentage is not available in our cohort, but may be a variable to be included in future studies.

\section{Conclusion}

An increased prevalence of overweight and obesity was observed in our pediatric post-transplant population, with older children at highest risk. In addition, patients without total body irradiation as part of the conditioning regimen experienced more weight gain than patients who received total body irradiation. Due to the well-documented negative effects of excess adiposity on health, it is crucial to optimize body composition during the pre-treatment, treatment, and post- treatment periods to mitigate the risk of catastrophic adverse events later in life. Additional studies characterizing the interaction between body composition and hematopoietic cell transplant outcomes are warranted. Greater insight into this field will undoubtedly provide guidance on the identification of at-risk populations and the development of interventions to treat and prevent obesity.

\section{Acknowledgements:}

This study was accomplished with funding from the St. Baldrick's Foundation.

\section{Competing Interests:}

All authors have no competing financial interests including products, devices, or drugs associated with this manuscript.

\section{References}

1. Styne DM, Arslanian SA, Connor EL, et al. Pediatric obesity-assessment, treatment, and prevention: An endocrine society clinical practice guideline. J Clin Endocrinol Metab . 2017. doi:10.1210/jc.2016-2573

2. Hruby A, Manson JAE, Qi L, et al. Determinants and consequences of obesity. Am J Public Health . 2016. doi:10.2105/AJPH.2016.303326

3. Pi-Sunyer X. The medical risks of obesity. Postgrad Med . 2009. doi:10.3810/pgm.2009.11.2074

4. Chan RSM, Woo J. Prevention of overweight and obesity: How effective is the current public health approach. Int J Environ Res Public Health . 2010. doi:10.3390/ijerph7030765

5. Garmey EG, Liu Q, Sklar CA, et al. Longitudinal changes in obesity and body mass index among adult survivors of childhood acute lymphoblastic leukemia: A report from the childhood cancer survivor study. $J$ Clin Oncol . 2008. doi:10.1200/JCO.2008.16.3527

6. Reilly JJ. Obesity during and after treatment for childhood cancer.Endocr Dev . 2009. doi:10.1159/000207609

7. Skoczen S, Surmiak M, Strojny W. Survivors of acute lymphoblastic leukemia and body mass changes. Expert Opin Drug Saf . 2010. doi:10.1517/14740330903410213 
8. Karaman S, Ercan O, Yildiz I, et al. Late effects of childhood ALL treatment on body mass index and serum leptin levels. J Pediatr Endocrinol Metab . 2010. doi:10.1515/JPEM.2010.23.7.669

9. Trimis G, Moschovi M, Papassotiriou I, Chrousos G, Tzortzatou-Stathopoulou F. Early indicators of dysmetabolic syndrome in young survivors of acute lymphoblastic leukemia in childhood as a target for preventing disease. J Pediatr Hematol Oncol . 2007. doi:10.1097/MPH.0b013e318059c249

10. van Waas M, Neggers SJCMM, Pieters R, van den Heuvel-Eibrink MM. Components of the metabolic syndrome in 500 adult long-term survivors of childhood cancer. Ann Oncol . 2009. doi:10.1093/annonc/mdp414

11. Geenen MM, Bakker PJM, Kremer LCM, Kastelein JJP, Leeuwen FEV. Increased prevalence of risk factors for cardiovascular disease in long-term survivors of acute lymphoblastic leukemia and Wilms tumor treated with radiotherapy. Pediatr Blood Cancer . 2010. doi:10.1002/pbc.22518

12. Zhang FF, Parsons SK. Obesity in Childhood Cancer Survivors: Call for Early Weight Management. Adv Nutr . 2015. doi:10.3945/an.115.008946

13. Copelan E. Hematopoietic Stem-Cell Transplantation. N Engl J Med . 2006;354(17):1813-1826.

14. Sorror ML, Maris MB, Storb R, et al. Hematopoietic cell transplantation (HCT)-specific comorbidity index: A new tool for risk assessment before allogeneic HCT. Blood . 2005. doi:10.1182/blood-2005-05-2004

15. Fleming DR, Rayens MK, Garrison J. Impact of obesity on allogeneic stem cell transplant patients: A matched case-controlled study. In:American Journal of Medicine .; 1997. doi:10.1016/S0002-9343(96)00450-0

16. White M, Murphy AJ, Hallahan A, Ware RS, Fraser C, Davies PSW. Survival in overweight and underweight children undergoing hematopoietic stem cell transplantation. Eur J Clin Nutr . 2012. doi:10.1038/ejcn.2012.109

17. Inaba H, Yang J, Kaste SC, et al. Longitudinal changes in body mass and composition in survivors of childhood hematologic malignancies after allogeneic hematopoietic stem-cell transplantation. J Clin Oncol . 2012. doi:10.1200/JCO.2011.40.0457

18. Ruble K, Hayat M, Stewart KJ, Chen A. Body composition after bone marrow transplantation in childhood. Oncol Nurs Forum . 2012. doi:10.1188/12.ONF.186-192

19. Child Growth Standards, WHO Anthro (version 3.1, June 2010) and macros. In: World Health Organization, 2010. pp macro calculate the indicators of the attained growth standards (length/height-for-age, weight-for-age, weight-for-length, weight-for-height, b.

20. A SAS Program for CDC Growth Charts. In: Center for Disease Control, 2009. p The purpose of this SAS program is to easily generate a dataset that contains indices of the anthropometric status of children from birth to 20 years of age based on the 2000 CDC.

21. Obesity: preventing and managing the global epidemic. Report of a WHO consultation. World Health Organ Tech Rep Ser. 2000;894:i-xii. 1-253.

22. Ogden CL, Carroll MD, Kit BK, Flegal KM. Prevalence of childhood and adult obesity in the United States, 2011-2012. JAMA - J Am Med Assoc . 2014. doi:10.1001/jama.2014.732

23. Los Angeles County Department of Public Health. Obesity and Related Mortality in Los Angeles County: A Cities and Communities Health Report .; 2011.

24. Cohen A, Duell T, Socié G, et al. Nutritional status and growth after bone marrow transplantation (BMT) during childhood: EBMT late-effects working party retrospective data. Bone Marrow Transplant . 1999. doi:10.1038/sj.bmt.1701769

25. Flowers MED, Martin PJ. How we treat chronic graft-versus-host disease. Blood . 2015. doi:10.1182/blood-2014-08-551994 
26. Jim HSL, Evans B, Jeong JM, et al. Sleep disruption in hematopoietic cell transplantation recipients: Prevalence, severity, and clinical management. Biol Blood Marrow Transplant . 2014. doi:10.1016/j.bbmt.2014.04.010

27. McBride B, Fiese B, Dev DA, et al. Risk factors for overweight/obesity in preschool children: An ecological approach. Child Obes . 2013. doi:10.1089/chi.2012.0150

28. Hjermstad MJ, Knobel H, Brinch L, et al. A prospective study of health-related quality of life, fatigue, anxiety and depression 3-5 years after stem cell transplantation. Bone Marrow Transplant . 2004. doi:10.1038/sj.bmt.1704561

29. Skaarud KJ, Veierød MB, Lergenmuller S, Bye A, Iversen PO, Tjønnfjord GE. Body weight, body composition and survival after 1 year: follow-up of a nutritional intervention trial in allo-HSCT recipients.Bone Marrow Transplant . 2019. doi:10.1038/s41409-019-0638-6

30. Monteiro R, Azevedo I. Chronic inflammation in obesity and the metabolic syndrome. Mediators Inflamm . 2010. doi:10.1155/2010/289645

31. Bubalo J, Carpenter PA, Majhail N, et al. Conditioning Chemotherapy Dose Adjustment in Obese Patients: A Review and Position Statement by the American Society for Blood and Marrow Transplantation Practice Guideline Committee. Biol Blood Marrow Transplant . 2014. doi:10.1016/j.bbmt.2014.01.019

32. Gleimer M, Li Y, Chang L, et al. Baseline body mass index among children and adults undergoing allogeneic hematopoietic cell transplantation: Clinical characteristics and outcomes. Bone Marrow Transplant . 2015. doi:10.1038/bmt.2014.280

33. Nakao M, Chihara D, Niimi A, et al. Impact of being overweight on outcomes of hematopoietic SCT: A meta-analysis. Bone Marrow Transplant . 2014. doi:10.1038/bmt.2013.128

34. Barker CC, Agovi MA, Logan B, et al. Childhood Obesity and Outcomes after Bone Marrow Transplantation for Patients with Severe Aplastic Anemia. Biol Blood Marrow Transplant . 2011. doi:10.1016/j.bbmt.2010.08.019

35. Couto-Silva AC, Trivin C, Esperou H, Michon J, Fischer A, Brauner R. Changes in height, weight and plasma leptin after bone marrow transplantation. Bone Marrow Transplant . 2000. doi:10.1038/sj.bmt.1702718

36. Shalitin S, Phillip M, Stein J, Goshen Y, Carmi D, Yaniv I. Endocrine dysfunction and parameters of the metabolic syndrome after bone marrow transplantation during childhood and adolescence. Bone Marrow Transplant . 2006. doi:10.1038/sj.bmt.1705374

37. Couto-Silva AC, Trivin C, Esperou H, et al. Final height and gonad function after total body irradiation during childhood. Bone Marrow Transplant . 2006. doi:10.1038/sj.bmt.1705455

38. Poirier P, Martin J, Marceau P, Biron S, Marceau S. Impact of bariatric surgery on cardiac structure, function and clinical manifestations in morbid obesity. Expert Rev Cardiovasc Ther . 2004. doi:10.1586/14779072.2.2.193

39. Poirier P, Giles TD, Bray GA, et al. Obesity and cardiovascular disease: Pathophysiology, evaluation, and effect of weight loss.Arterioscler Thromb Vasc Biol . 2006. doi:10.1161/01.ATV.0000216787.85457.f3

40. Chatterjee K, Zhang J, Honbo N, Karliner JS. Doxorubicin cardiomyopathy. Cardiology . 2010. doi:10.1159/000265166

41. Warburg O. On the origin of cancer cells. Science (80- ) . 1956. doi:10.1126/science.123.3191.309

42. Tan-Shalaby J. Ketogenic Diets and Cancer: Emerging Evidence.Fed Pract . 2017.

43. Di Biase S, Longo VD. Fasting-induced differential stress sensitization in cancer treatment. Mol Cell Oncol . 2016;3(3):1-2. doi:10.1080/23723556.2015.1117701 
44. Longo VD, Fontana L. Calorie restriction and cancer prevention: metabolic and molecular mechanisms. Trends Pharmacol Sci . 2010. doi:10.1016/j.tips.2009.11.004

45. Adlercreutz H. Western diet and western diseases: Some hormonal and biochemical mechanisms and associations. Scand J Clin Lab Invest . 1990. doi:10.1080/00365519009085798

46. Kuriki K, Tajima K. The increasing incidence of colorectal cancer and the preventive strategy in Japan. Asian Pacific J Cancer Prev . 2006.

47. Craig WJ. Health effects of vegan diets. In: American Journal of Clinical Nutrition . ; 2009. doi:10.3945/ajcn.2009.26736N

48. Schwingshackl L, Hoffmann G. Adherence to Mediterranean diet and risk of cancer: An updated systematic review and meta-analysis of observational studies. Cancer Med . 2015;4(12):1933-1947. doi:10.1002/cam4.539

49. Gray A, Dang BN, Moore TB, Clemens RA, Pressman P. A Review of Nutrition and Dietary Interventions in Oncology. SAGE Open Med .

\section{Figure Legends}

\section{FIGURE 1 BMI trends over time stratified by age}

Plot of the BMI changes relative to baseline (pre-transplant), starting from day 100 to 5 years post-transplant. Day 100 BMI was not significantly different from baseline BMI for any age. All ages demonstrated significant increases in BMI over time from day 100 to 5 years post-transplant (all $p<0.001$ ).

\section{FIGURE 2 Survival estimates}

Kaplan-Meier plot of overall survival probability in HCT patients.

\section{Hosted file}

Table 1.docx available at https://authorea.com/users/328908/articles/456040-pediatrichematopoietic-cell-transplantation-longitudinal-trends-in-body-mass-index-and-outcomes

\section{Hosted file}

Table 2.docx available at https://authorea.com/users/328908/articles/456040-pediatrichematopoietic-cell-transplantation-longitudinal-trends-in-body-mass-index-and-outcomes

\section{Hosted file}

Table 3.docx available at https://authorea.com/users/328908/articles/456040-pediatrichematopoietic-cell-transplantation-longitudinal-trends-in-body-mass-index-and-outcomes 


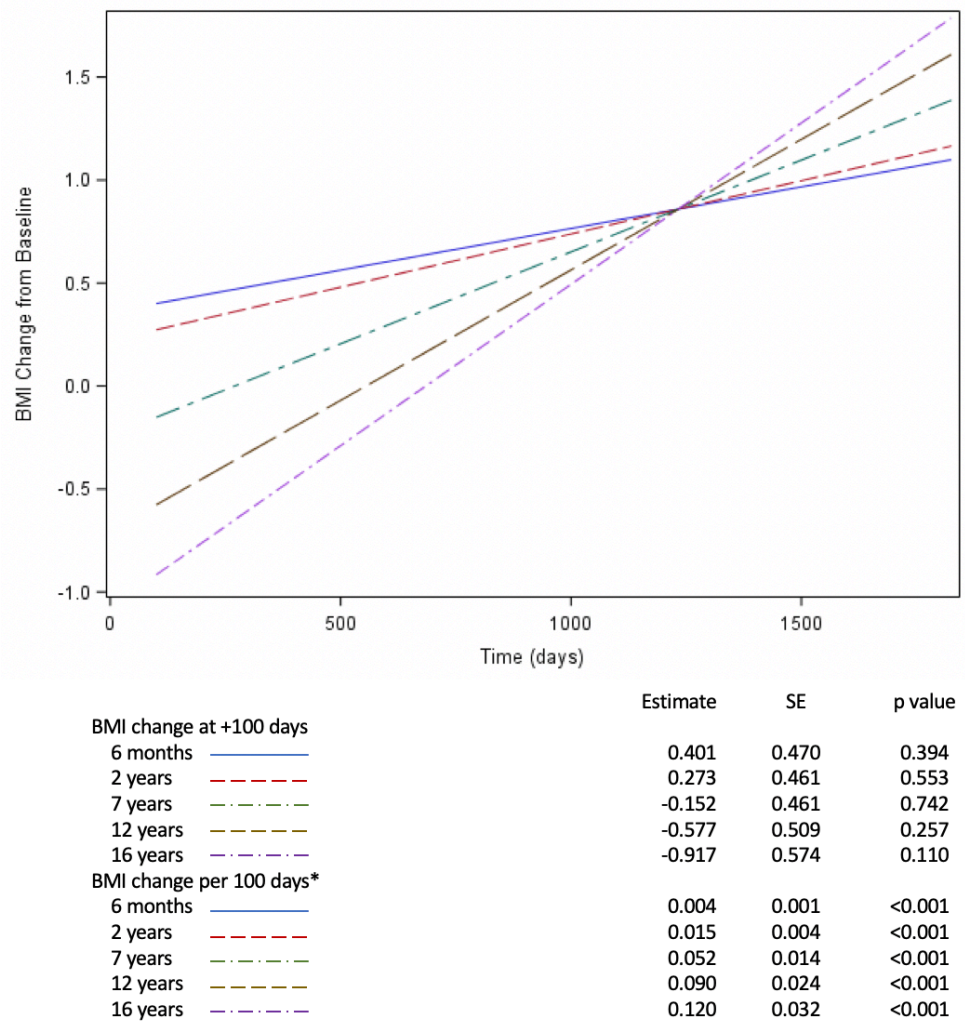




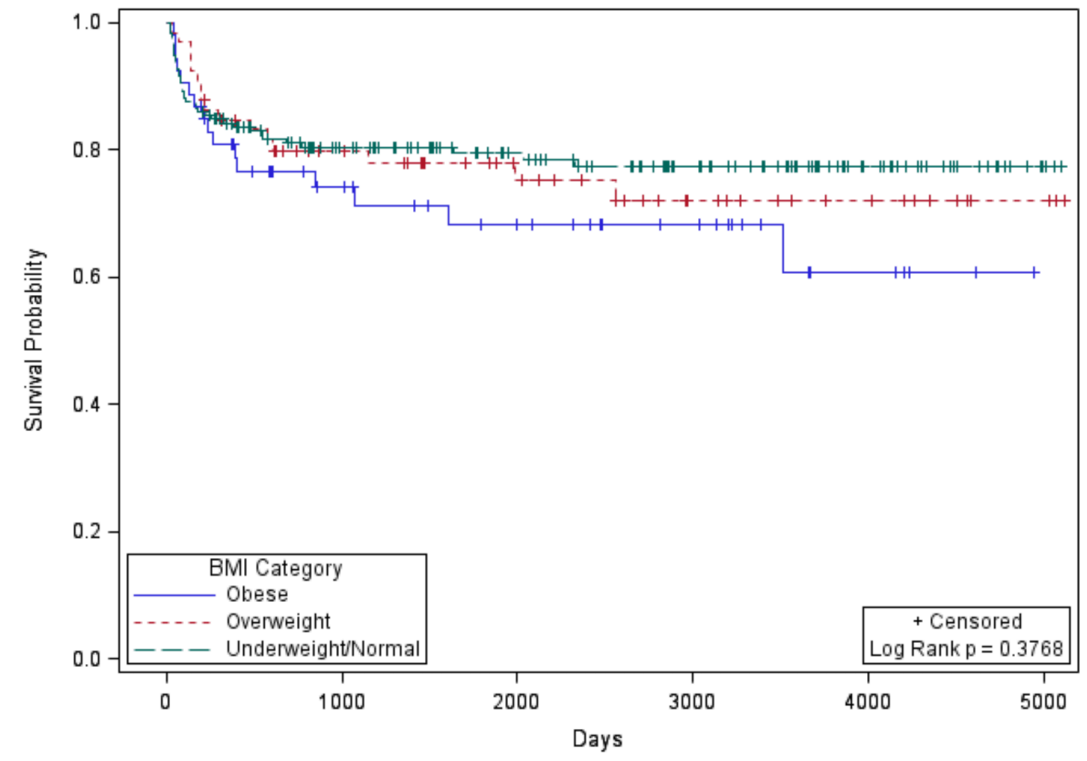

